\title{
On the Generalization of the Timoshenko Beam Model Based on the Micropolar Linear Theory: Static Case
}

\author{
Andrea Nobili \\ Dipartimento di Ingegneria "Enzo Ferrari”, via Vignolese 905, 41125 Modena, Italy \\ Correspondence should be addressed to Andrea Nobili; andrea.nobili@unimore.it
}

Received 3 December 2014; Revised 25 February 2015; Accepted 26 February 2015

Academic Editor: Efstratios Tzirtzilakis

Copyright (C) 2015 Andrea Nobili. This is an open access article distributed under the Creative Commons Attribution License, which permits unrestricted use, distribution, and reproduction in any medium, provided the original work is properly cited.

\begin{abstract}
Three generalizations of the Timoshenko beam model according to the linear theory of micropolar elasticity or its special cases, that is, the couple stress theory or the modified couple stress theory, recently developed in the literature, are investigated and compared. The analysis is carried out in a variational setting, making use of Hamilton's principle. It is shown that both the Timoshenko and the (possibly modified) couple stress models are based on a microstructural kinematics which is governed by kinosthenic (ignorable) terms in the Lagrangian. Despite their difference, all models bring in a beam-plane theory only one microstructural material parameter. Besides, the micropolar model formally reduces to the couple stress model upon introducing the proper constraint on the microstructure kinematics, although the material parameter is generally different. Line loading on the microstructure results in a nonconservative force potential. Finally, the Hamiltonian form of the micropolar beam model is derived and the canonical equations are presented along with their general solution. The latter exhibits a general oscillatory pattern for the microstructure rotation and stress, whose behavior matches the numerical findings.
\end{abstract}

\section{Introduction}

One truly remarkable feature of the theory of elasticity is the possibility of dealing with the vast microscopic complexity and diversity of real materials in a unified and "averaged" fashion. Such feature is best illustrated by the broad class of linear isotropic materials, which can be described from a mechanical standpoint by means of just two material parameters. Of course, this far standing attitude cannot be expected to work just as well when very small devices, whose size is in the order of the microstructure length scale, are considered. To overcome such short-coming and to effectively model the current trend of micro- and nanoelectromechanical systems, MEMS and NEMS, several nonclassical continuum theories have been developed. Among these, we will mention the micropolar continuum theory $[1,2]$, hereinafter referred to as the Eringen-Nowacki (E-N) micropolar theory [3], which adds at each material point a microstructure description in terms of one rotation vector. In general, the adoption of a nonclassical continuum theory takes a heavy toll in that either extra boundary conditions are demanded or a considerable number of material parameters need to be somehow determined. For the case of the E-N micropolar theory, three microstructural material parameters are required. Consequently, one very desirable feature of a workable nonclassical continuum theory is the ability to capture the mechanical bearing of the microstructure at the macroscale at the least possible cost in terms of experimental effort for the determination of the material parameters. The desire to strike this difficult balance has spawn a number of specialized versions of the micropolar theory, such as the Koiter-Mindlin (K-M) couple stress theory $[4,5]$ and, recently, the reduced couple stress theory [6], wherein, respectively, two and one material parameters are introduced. When such diverse models are confronted with approximate theories, such as the beam's, generally unexpected results are in order [7]. The modified couple stress theory has been applied to develop an EulerBernoulli model in [8]. However, attention in the literature is mostly focused on Timoshenko-like models, in light of their advantages. Indeed, the Timoshenko model incorporates the shear contribution to the deformation, which is most relevant in the thick-beam high-frequency regime proper of MEMS. 


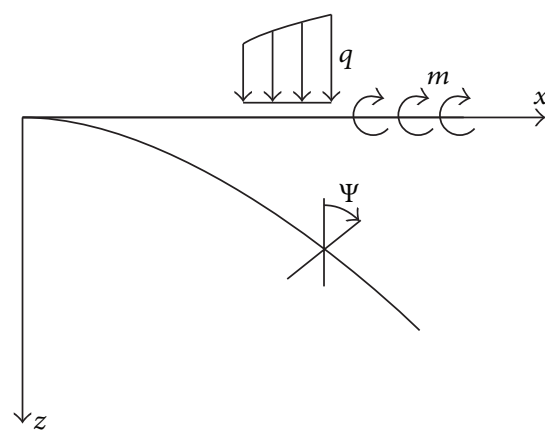

(a)

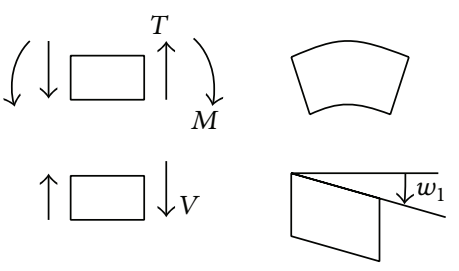

(b)

FIGURE 1: Kinematics of a Timoshenko beam (a) and positivity conventions (b).

Besides, the Timoshenko model is variationally more apt to a FE approximation (no locking effect). Plates and rod models are mathematically justified through a proper scaling in a small parameter from the three-dimensional theory of linear micropolar elasticity in $[9,10]$. A Timoshenko beam model based on the reduced couple stress theory is presented in [11], where static bending and free vibration of a simply supported beam are studied. In [12], a First Order Shear Deformation Beam Theory (FSDBT) is developed for micropolar elastic beams and analytical results for the static bending of a cantilever beam as well as for the dispersion relation for longitudinal and flexural waves are given. Couple stress Timoshenko beams have been investigated in [13], where a closed-form solution valid in the static case and for homogeneous materials is obtained and then applied to analyze a cantilever beam. In a such a variety, the question of whether such models reconcile and under what conditions seldom appears and when it does the answer rests in the negative. This paper attempts to address precisely this matter and to gather all models under a single variational framework. The issue of what assumptions really matter for the reduction in the number of material parameters is also investigated. The paper is thus organized. Section 2 revises some feature of the Timoshenko beam model, Section 3 introduces the couple stress Timoshenko model, and Section 4 introduces the broader linear micropolar model. The Hamiltonian form of the latter and its general solution are presented in Section 4.1. Finally, conclusions are drawn in Section 5.

\section{Variational Features of the Timoshenko Beam}

Let us consider a prismatic body (beam) of cross-sectional area $A$ and length $L$, whose central axis (the sections' centroid line) rests along the $x$-axis in the reference configuration, Figure 1. Traditionally, a Timoshenko beam [14, Section 2.17] is characterized by the following Lagrangian density:

$$
\mathscr{L}=\frac{1}{2} E I \psi_{1}^{2}+\frac{1}{2} A G\left(y_{1}-\psi\right)^{2}-q y-m \psi,
$$

where $E I$ is the beam flexural rigidity, $G$ is the shear modulus, $y$ is the transverse displacement, $\psi$ is the cross-section rotation (positive clockwise), and $q$ and $m$ are the transverse and the torque line load density, respectively. (This notation is Timoshenko's and we abide by it for historical reasons. The notation $z$ for the transverse displacement would better fit with the reference system of Figure 1, which is commonly adopted in the recent literature. However, confusion may arise with the $z$ coordinate along the cross-section.) Since this is a beam theory, all field quantities depend on the single independent variable $x$, that is, $E I=E I(x), q=q(x)$, and so forth. Here, we use a subscript number to denote differentiation with respect to $x$; that is, we use $y_{1}$ in the place of $d y / d x$. Besides, we will restrict ourselves to the static theory. For the sake of notational compactness, the shear factor $k_{s}$, usually associated with the Timoshenko model, is here omitted, although it can be easily introduced whenever a factor $A G$ appears. The action integral is

$$
\mathscr{A}=\int_{0}^{L} \mathscr{L} d x
$$

and according to Hamilton's principle seeking for deformation makes the action integral stationary. Since here the axial coordinate $x$ takes up the role which is usually played by time $t$ in discrete mechanics, we will refer to $y_{1}$ as the velocity and to $y_{2}$ as the acceleration. The Euler-Lagrange (E-L) equations for the Lagrangian (1) are

$$
\begin{array}{r}
\left(E I \psi_{1}\right)^{\prime}+A G\left(y_{1}-\psi\right)+m=0, \\
{\left[A G\left(y_{1}-\psi\right)\right]^{\prime}+q=0,}
\end{array}
$$

where prime denotes differentiation with respect to $x$.

The Lagrangian (1) is noteworthy because it contains the gyroscopic term $\psi y_{1}$, that is, a term which is linear in the velocity. It is well known that a gyroscopic term arises from the elimination of kinosthenic (or ignorable) variables $[15$, Chapter V, Section 4]. Indeed, we easily show that the Lagrangian (1) is obtained from the enlarged Lagrangian:

$$
\mathscr{L}=\frac{1}{2} E I \psi_{1}^{2}+\frac{1}{2} A G w_{1}^{2}-q y-m \psi-\mu\left(y_{1}-w_{1}-\psi\right) .
$$

Here, $w_{1}$ is the slope that arises from shear deformation (see Figure 1(b)), on account of the shearing force $V=$ $A G w_{1}$. On the other hand, the total slope $y_{1}$ is partly due to 
the rigid rotation of the cross-section, $\psi$, and partly due to the shear deformation, $w_{1}$. The last term of (4) stems from such kinematical connection and its dealing through the Lagrange multiplier method. Nonetheless, the variational principles (1) and (4) are not entirely equivalent. Indeed, the E-L equations of (4) are

$$
\begin{aligned}
\left(E I \psi_{1}\right)^{\prime}-\mu+m & =0 \\
\mu^{\prime}-q & =0 \\
\left(A G w_{1}+\mu\right)^{\prime} & =0
\end{aligned}
$$

together with the constraint

$$
y_{1}=w_{1}+\psi
$$

Comparing such equations with the rotational and vertical equilibrium equations, respectively,

$$
\begin{array}{r}
M^{\prime}-T+m=0, \\
T^{\prime}-q=0,
\end{array}
$$

shows that $\mu=T$ is the shearing force acting on a crosssection and participates with the bending moment $M=$ $E I \psi_{1}$ to rotational equilibrium. It is emphasized that only the shearing force $V$ causes shear deformation $\gamma=V /(A G)$, while the shearing force $T$ is the traditional shearing force associated with a Euler-Bernoulli model; that is, it causes no direct deformation. So, it can be seen that this model is more general than the traditional Timoshenko beam, for in it a shearing force $V$ exists which causes shear deformation and yet it is associated with no cross-section rotation; that is, the bending moment required to warrant rotational equilibrium appears at no energy cost. In such a model, the pure shear deformation with no cross-section rotation shown at the bottom right corner of Figure 1 may exist on its own right. Only through the boundary conditions (BCs), the two models may be entirely reconciled. Indeed, as it is the case with kinosthenic variables, (5c) may be immediately integrated giving

$$
A G w_{1}+\mu=V+T=\bar{V},
$$

where $\bar{V}$ is a constant and it equals the boundary shearing force conjugated with $\delta w$. If $\bar{V}=0$, then $T=-V$ and the shearing force $T$ determines both shear deformation and cross-section rotation at the same time; that is, a pure shear deformation is impossible. This is the Timoshenko model, where only two natural BCs appear, namely,

$$
\left(E I \psi_{1}\right) \delta \psi+A G\left(y_{1}-\psi\right) \delta y .
$$

However, if we were to consider $\bar{V}$ different form zero, we would allow for a distinction between the shearing forces $T$ and $V$. Indeed, in this general case, we would have three natural boundary conditions, namely,

$$
\left(E I \psi_{1}\right) \delta \psi-\mu \delta y+\bar{V} \delta w .
$$

This difference in the BCs is a major issue when dealing with free boundary problems $[16,17]$.

\section{The Couple Stress Timoshenko Beam}

Many generalizations of the Timoshenko beam have been recently proposed in the literature, which are based on the micropolar linear continuum theory of Eringen-Nowacki (EN) [1] or its special cases, the couple stress theory of KoiterMindlin [5] and the reduced couple stress theory [6], in an attempt to incorporate a scale effect in the theory. In the E-N model, a microstructure exists whose single degree of freedom with respect to the macrostructure is the rotation (a similar situation occurs in electro- or magneto-elastic materials, where a vectorial microstructure is considered [18-20]), described by the microstructure pseudorotation vector $\boldsymbol{\theta}$ (pseudo because it reverses sign upon passing from a right-handed to a left-handed reference system). Micro- and macrostructure share the same macromotion, described by the displacement field $\mathbf{u}$. In the special case of the KoiterMindlin (K-M) couple stress theory, the microstructure rotation is no longer an independent degree of freedom but rather it is bound to the macrostructure deformation; that is, it is a latent microstructure. Consequently [5, Eq. (2.17)],

$$
\boldsymbol{\theta}=\frac{1}{2} \operatorname{curl} \mathbf{u}
$$

where, componentwise, $(\operatorname{curl} \mathbf{u})_{i}=\epsilon_{i j k} \partial u_{j} / \partial x_{k}$ and $\epsilon_{i j k}$ is the permutation symbol or alternator. Since we are here considering a plane beam theory, every motion takes place in the $(x, z)$-plane and we let

$$
\begin{aligned}
& \mathbf{u}=\left[\begin{array}{lll}
u_{x} & 0 & u_{z}
\end{array}\right], \\
& \boldsymbol{\theta}=\left[\begin{array}{lll}
0 & \varphi & 0
\end{array}\right],
\end{aligned}
$$

being

$$
\begin{aligned}
& u_{x}=u_{x}(x, z), \\
& u_{z}=y(x), \\
& \varphi=\varphi(x) .
\end{aligned}
$$

Note that here $\varphi$ is positive when being counterclockwise (following the right hand screw rule about the $y$-axis), as opposed to $\psi$, which is positive when being clockwise (Figure 1). In a Timoshenko-like theory, the cross-section originally at coordinate $x$ remains plane after deformation, whence

$$
u_{x}=u_{0}-z \psi,
$$

which is sometimes referred to as a Timoshenko's First Order Shear Deformation Beam Theory (FSDBT) [12, 21]. More general theories can be constructed by considering the general power series expansion along $z$ [21]:

$$
u_{x}(x, z)=\sum_{i=0}^{N} z^{i} \psi_{(i)}(x)
$$

of which (14) corresponds to the case $N=1$. For simplicity, we will neglect the axial extension; that is, $u_{0}=0$, given that 
it turns out to be decoupled from the transverse deformation. Again, this is a beam theory and all field variables $\psi$ and $y$ are functions of $x$. Letting the strain energy for the beam occupy the three-dimensional prismatic region $\Omega$ [5, Eq. (2.25)],

$$
U=\frac{1}{2} \int_{\Omega}\left(\boldsymbol{\sigma} \cdot \boldsymbol{\epsilon}+\mathbf{m}^{*} \cdot \boldsymbol{\kappa}\right) d v
$$

where $\boldsymbol{\sigma}$ is the Cauchy stress tensor, $\boldsymbol{\epsilon}=\operatorname{Sym}[\operatorname{grad} \mathbf{u}]$ is the linear strain tensor, $\mathbf{m}^{*}$ is the couple stress tensor, and $\boldsymbol{\kappa}$ is the microstrain tensor (also termed torsion-flexure or wryness). (A concise account of the E-N model and other micropolar theories can be found in the recent monograph [3]. As far as the notation goes, the asymmetric strain tensor $\boldsymbol{\gamma}$ and the microstrain tensor $\boldsymbol{\kappa}$ are the transpose of our $\mathbf{e}$ and $\boldsymbol{\kappa}$, respectively, given that div and grad are also defined in a "transposed" way.) Consider

$$
\boldsymbol{\kappa}=\operatorname{grad} \boldsymbol{\theta} .
$$

The symmetric part of the wryness tensor is the curvature tensor:

$$
\boldsymbol{\chi}=\operatorname{Sym}[\operatorname{grad} \boldsymbol{\theta}]=\frac{1}{2}\left(\operatorname{grad} \boldsymbol{\theta}+\operatorname{grad}^{T} \boldsymbol{\theta}\right) .
$$

Here, the superscript $T$ denotes transposition and, componentwise, $(\operatorname{grad} \boldsymbol{\theta})_{i j}=\partial \theta_{i} / \partial x_{j}$. Besides, a dot denotes scalar product; namely, $\boldsymbol{\sigma} \cdot \boldsymbol{\epsilon}=\sigma_{i j} \epsilon_{i j}$. The usual constitutive equation for linear elastic isotropic homogeneous material is assumed as

$$
\boldsymbol{\sigma}=\lambda(\operatorname{tr} \boldsymbol{\epsilon}) \mathbf{1}+2 G \boldsymbol{\epsilon}
$$

where $\mathbf{1}$ is the identity tensor and $\lambda, G$ are Lamé constants. In light of (11)-(14), it is (cf. [11, Eq. (10)])

$$
\begin{aligned}
& \epsilon_{x x}=-z \psi_{1}, \\
& \epsilon_{x z}=\epsilon_{z x}=\frac{1}{2}\left(y_{1}-\psi\right)
\end{aligned}
$$

and $\epsilon_{y y}=\epsilon_{z z}=\epsilon_{x y}=\epsilon_{y z}=0$. Again (11)-(14) give the connection between the microstructure rotation vector $y$-component and the cross-section macrorotation [11, Eq. (11)]:

$$
\varphi=-\frac{1}{2}\left(\psi+y_{1}\right)
$$

Consequently, the only nonzero component of the wryness tensor is

$$
\kappa_{y x}=\varphi_{1}
$$

while the only nonzero components of the curvature tensor are

$$
\chi_{x y}=\chi_{y x}=\frac{1}{2} \varphi_{1}
$$

Letting the couple stress tensor $\mathbf{m}^{*}$ be conjugated to the wryness tensor $\boldsymbol{\kappa}$, through the constitutive assumption (2) of [13],

$$
\mathbf{m}^{*}=\alpha(\operatorname{div} \boldsymbol{\theta}) \mathbf{1}+\gamma^{*} \operatorname{grad} \boldsymbol{\theta}+\beta^{*} \operatorname{grad}^{T} \boldsymbol{\theta}
$$

wherein $\alpha, \beta^{*}$ and $\gamma^{*}$ are three material constants. Here, $\operatorname{div} \boldsymbol{\theta}=\operatorname{tr}(\operatorname{grad} \boldsymbol{\theta})$ or, componentwise, $\operatorname{div} \boldsymbol{\theta}=\partial \theta_{i} / \partial x_{i}$, and summation over repeated indexes is implied. In light of the constraint (11), it is $\operatorname{div} \boldsymbol{\theta}=0$. We then see that in the couple stress theory the material constants pertaining to the microstructure are reduced to two. Furthermore, by the same constraint, the spherical part of $\boldsymbol{\kappa}$ is zero (i.e., its first invariant vanishes, $\operatorname{tr} \boldsymbol{\kappa}=0$ ), whence the spherical part of the couple stress tensor rests undetermined [5]. Equations (22) and (24) give

$$
m_{x y}^{*}=\beta^{*} \varphi_{1}
$$

In the case of the modified couple stress theory, only the symmetric part of $\mathbf{m}^{*}$ is considered, thus further reducing the number of material constants to just one [11]; that is,

$$
\mathbf{m}=\operatorname{Sym}\left[\mathbf{m}^{*}\right]=2 \beta \chi,
$$

where $2 \beta=\gamma^{*}+\beta^{*}$. Equations (23) and (26) yield

$$
m_{x y}=2 \beta \chi_{x y}=\beta \varphi_{1}
$$

and regardless of whether the couple stress or the modified couple stress theory is adopted only one constitutive parameter appears for the $x y$-couple stress component, respectively, $\beta^{*}$ or $\beta$. When the constitutive assumption (26) is compared with (8) of [11], namely,

$$
\mathbf{m}=2 l^{2} G \chi
$$

it is clearly seen that the single material parameter, $\beta$, originating from the couple stress theory, is taken proportional to the shear module $G$ through the material characteristic length $l$. Besides, it is noted that the bending moment $m_{y x}$ appears owing to the microstructure, which requires a specific constraint to dispense with the out-of-plane deformation this would cause.

It is now possible to write the Lagrangian density whose E-L equations are (17) and (18) of [13]; namely,

$$
\begin{aligned}
\mathscr{L}= & \frac{1}{2} E I \psi_{1}^{2}+\frac{1}{2} A G\left(y_{1}-\psi\right)^{2}+\frac{1}{8} \beta A\left(\psi_{1}+y_{2}\right)^{2} \\
& -q y-m \psi .
\end{aligned}
$$

This Lagrangian density is obtained integrating (16) along the cross-section, lying in the $(y, z)$ plane, having introduced (19)-(26) together with the restricted kinematics (11), (12) [11]. The result accounts for the first three terms at RHS of (29), whereas the last two terms convey the potential of the external loads. It is observed that a quadratic term in the acceleration $y_{2}$ appears in the Lagrangian (29); that is, we have an acceleration dependent Lagrangian. Furthermore, an extra gyroscopic-like term exists, which is linear in the acceleration, namely, $\psi_{1} y_{2}$. The E-L equations are

$$
\begin{array}{r}
{\left[E I \psi_{1}+\frac{1}{4} \beta A\left(\psi_{1}+y_{2}\right)\right]^{\prime}+A G\left(y_{1}-\psi\right)+m=0,} \\
\left\{\left[\frac{1}{4} \beta A\left(\psi_{1}+y_{2}\right)\right]^{\prime}-A G\left(y_{1}-\psi\right)\right\}^{\prime}-q=0,
\end{array}
$$




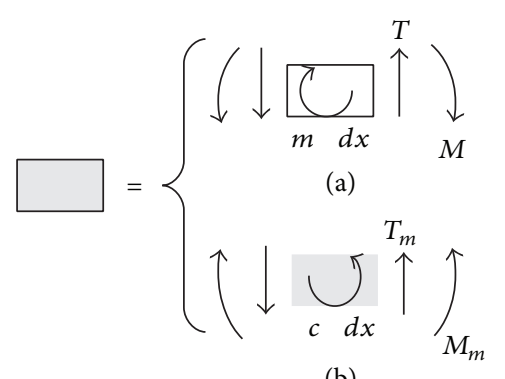

(b)

Figure 2: Actions (a) on the macro- and (b) on the microstructure.

which are the self-adjoint form (i.e., the generalization to inhomogeneous materials) of the static version of (17) and (18) in [13]. (It needs to be considered that $w$ and $\omega$ there are the counterparts of our $-y$ and $-q$, given that the $z$-axis is oriented upwards; that is, $w \mapsto-y$ and $\omega \mapsto-q$. Besides, $\psi \mapsto-\psi$.) Equations (30a) and (30b) may be rewritten in the form of an equilibrium equation set (cf. (7)):

$$
\begin{aligned}
\left(M-M_{m}\right)^{\prime}+V+m & =0, \\
\left(T_{m}-V\right)^{\prime}-q & =0,
\end{aligned}
$$

wherein $M_{m}=-(1 / 4) \beta A\left(\psi_{1}+y_{2}\right)$ is the bending moment brought in by the microstructure (cf. (23) of [11]), which conveys the shearing force $T_{m}=-M_{m}^{\prime}$. It is observed that the microstructure bending moment, $M_{m}$, and shearing force, $T_{m}$, are positive according to the convention of Figure 2 .

We will now show how, from a mechanical standpoint, the couple stress theory appears in a new enlarged Lagrangian, wherein the microstructure rotation is given the status of dependent variable. The Lagrangian (29) may be rewritten as

$$
\begin{aligned}
\mathscr{L}= & \frac{1}{2} E I \psi_{1}^{2}+\frac{1}{2} A G\left(y_{1}-\psi\right)^{2}+\frac{1}{2} \beta A \varphi_{1}^{2}-q y-m \psi \\
& -c \varphi+\lambda\left(2 \varphi+\psi+y_{1}\right),
\end{aligned}
$$

having introduced the Lagrangian multiplier $\lambda$ and the distributed torque $c$ acting upon the microstructure (positive when counterclockwise, cf. Figure 2). The third term in (32) may be reconciled with the couple stress theory in light of (27). The Lagrangian (32) gives the following E-L equations:

$$
\begin{aligned}
\left(E I \psi_{1}\right)^{\prime}+A G\left(y_{1}-\psi\right)-\lambda+m & =0, \\
-\left[A G\left(y_{1}-\psi\right)+\lambda\right]^{\prime}-q & =0, \\
\left(\frac{1}{2} \beta A \varphi_{1}\right)^{\prime}-\lambda+\frac{1}{2} c & =0,
\end{aligned}
$$

together with the constraint (21). Recalling the general form of the equilibrium equation (7), (33c) may be interpreted as giving the rotational equilibrium of the microstructure, which is acted upon by the bending moment $M_{m}=(1 / 2) \beta A \varphi_{1}$ and the distributed torque $c / 2-\lambda$, while the shearing force $T_{m}=$ 0 . Equation (33a) enforces the rotational equilibrium of the macrostructure:

$$
M^{\prime}+V+m-\lambda=0,
$$

which shows that the distributed torque $\lambda$ is exchanged with the microstructure according to the action-reaction principle. Finally, (33b) is the vertical equilibrium for the macrostructure, which is acted upon by the shearing force $V=$ $A G\left(y_{1}-\psi\right)$ and the shearing force $T=-\lambda$. This mechanical interpretation of the equations, where the distributed torque $\lambda$ appears also as a shearing force, derives from the form of the last term in the Lagrangian (32), wherein $\lambda$ is conjugated to the rotation $\varphi$, like $c$, and to the shear-like term $y_{1}+\psi$, like $V$. (It is observed that the Lagrange multiplier $\lambda$ may be given yet another mechanical interpretation: the distributed torque $\lambda$ is mechanically equivalent to a line load distribution $q_{m}=\lambda^{\prime}$, in a manner similar to the dealing with that boundary condition for the twisting moment in the Kirchhoff-Love plate theory. However, unlike the twisting moment in the Kirchhoff-Love plate theory, here $\lambda$ appears in the equations in two forms, i.e., both as the line torque $\lambda$ and as the line load $\lambda^{\prime}$.)

We now consider the special case $c=0$ and modify the Lagrangian (32) so that $\varphi_{1}$ is kinosthenic:

$$
\begin{aligned}
\mathscr{L}= & \frac{1}{2} E I \psi_{1}^{2}+\frac{1}{2} A G\left(y_{1}-\psi\right)^{2}+\frac{1}{2} \beta A \varphi_{1}^{2}-q y-m \psi \\
& +\mu\left(2 \varphi_{1}+\psi_{1}+y_{2}\right) .
\end{aligned}
$$

Note that this step reintroduces an acceleration term and that $4 \mu$ is now conjugated with the curvature $\varphi_{1} / 2$ just like the microstructure bending moment $M_{m}$. As a result, this new Lagrangian affords a simple mechanical interpretation. The new E-L equations are

$$
\begin{aligned}
\left(E I \psi_{1}+\mu\right)^{\prime}+A G\left(y_{1}-\psi\right)+m & =0, \\
\mu^{\prime \prime}-\left[A G\left(y_{1}-\psi\right)\right]^{\prime}-q & =0, \\
\left(\frac{1}{2} \beta A \varphi_{1}+\mu\right)^{\prime} & =0,
\end{aligned}
$$

plus the derivative of the constraint (21), which determines $\varphi$ as a function of $y_{1}+\psi$ up to a constant. (This indeterminacy is the reason why we need consider the case $c=0$.) In this new variational setting, $\mu$ is the exchange bending moment between the macro- and the microstructure, the latter now receiving no exchange distributed torque. The exchange bending moment participates in the vertical equilibrium equation (36b) in the form of a shearing force $T=\mu^{\prime}$. Since $\varphi_{1}$ is now kinosthenic, it can be eliminated giving the Lagrangian (29), in a similar manner as with the Timoshenko model. Indeed, integrating (36c) gives

$$
M_{m}+\mu=\bar{M}_{m}
$$

where $\bar{M}_{m}$ is the BC imposing the microstructure bending moment. When $\bar{M}_{m}=0$, we have $\mu=-M_{m}=(1 / 4) \beta A\left(\psi_{1}+\right.$ $\left.y_{2}\right)$ and (36a) and (36b) turn into (30a) and (30b), respectively.

\section{The E-N Micropolar Timoshenko Beam}

When the E-N micropolar linear theory is employed, the microstructure rotation $\boldsymbol{\theta}$ is no longer bound to the macrostructure rotation and, in particular, the constraint (21) 
drops out. As a consequence, the constitutive equation (24) may be applied in full form:

$$
\mathbf{m}^{*}=\alpha(\operatorname{div} \boldsymbol{\theta}) \mathbf{1}+\gamma^{*} \boldsymbol{\kappa}+\beta^{*} \boldsymbol{\kappa}^{T},
$$

which shows that three constitutive parameters come in from the microstructure. Furthermore, the micropolar theory entails that the stress tensor $\boldsymbol{\sigma}^{*}$ is no longer symmetric and its skew-symmetric part, $\operatorname{Skw}\left[\boldsymbol{\sigma}^{*}\right]$, is energy conjugated with the macrostructure rotation; that is, it does provide an energy contribution. Letting the linear micropolar strain tensor,

$$
\begin{aligned}
\mathbf{e} & =\operatorname{grad} \mathbf{u}+\mathbf{\Omega}, \\
\Omega_{i j} & =\epsilon_{i j k} \theta_{k},
\end{aligned}
$$

where $\boldsymbol{\Omega}$ is the skew-symmetric tensor associated with the rotation vector $\boldsymbol{\theta}$; that is, the latter is the axial vector of the former. The constitutive equation now needs to specify the full stress tensor; namely,

$$
\boldsymbol{\sigma}^{*}=\lambda(\operatorname{tr} \mathbf{e}) \mathbf{1}+2 G \operatorname{Sym}[\mathbf{e}]+\eta \operatorname{Skw}[\mathbf{e}] .
$$

Obviously, $\lambda$ and $G$ are the usual Lamé constants, which specify the symmetric part of the stress tensor, while $\eta$ determines the skew symmetric part. The Lagrangian density corresponding to the E-L equations (35)-(37) of [12] is

$$
\begin{aligned}
\mathscr{L}= & \frac{1}{2} E I \psi_{1}^{2}+\frac{1}{2} A G\left(y_{1}-\psi\right)^{2}+\frac{1}{2} \beta^{*} A \varphi_{1}^{2} \\
& -\frac{1}{4} \eta A\left(y_{1}+\psi\right)^{2}-q y-m \psi-c \varphi \\
& -A \eta \varphi\left(\varphi+y_{1}+\psi\right) .
\end{aligned}
$$

This Lagrangian has been obtained integrating along the beam cross-section the strain energy

$$
U^{*}=\frac{1}{2} \int_{\Omega}\left(\boldsymbol{\sigma}^{*} \cdot \mathbf{e}+\mathbf{m}^{*} \cdot \boldsymbol{\kappa}\right) d v
$$

and accounting for the external load potential. Introducing the constraint (21) and assuming the special case $c=0$, the fourth term and the last term cancel each other and we are left with the Lagrangian density (35). Indeed, this result may be at the root of the equivalence observed in [8] for the deflection expression of a cantilever beam within the modified couple stress and the micropolar theory. However, attention should be drawn to the fact that the material constant $\beta^{*}$ appears instead of $\beta=\left(\beta^{*}+\gamma^{*}\right) / 2$. Besides, the last of (12) and the beam theory assumptions (13) give

$$
\operatorname{div} \boldsymbol{\theta}=0
$$

whence the material parameter $\alpha$ never appears. Indeed, in this plane deformation, only the microstructural constitutive parameter $\beta^{*}$ plays a role. In the general case $c \neq 0$, the external load potential appears as

$$
\frac{1}{2} c\left(y_{1}+\psi\right)
$$

This loading potential provides us with an example of a monogenic yet nonconservative loading [15, Chapter I, Section 7], just like the case of a follower force. It is then seen that the E-N micropolar beam model and the couple stress beam model formally reconcile, although they actually coincide only in the very special case when $c \equiv 0$ and $\gamma^{*} \equiv \beta^{*}$. Furthermore, the case $\eta=0$ is especially interesting because then the E-N model and the couple stress model formally coincide apart from the last term of (35). The latter disappears when neither exchange distributed torque $\mu$ acts nor the constraint (21) holds. Obviously, in the case $\eta \equiv 0$, the macroand the microstructure equilibrium equations decouple and the former reduce to the Timoshenko model. Clearly, the further assumption $A G \rightarrow \infty$ lends the Euler-Bernoulli model.

4.1. Hamiltonian Form and General Solution. For the E-N model, we now derive the Hamiltonian form and the corresponding canonical equations. Following [16], let the generalized momenta

$$
\begin{aligned}
& p_{1}=A G\left(y_{1}-\psi\right)-\frac{1}{2} \eta A\left(y_{1}+\psi\right)-A \eta \varphi, \\
& p_{2}=E I \psi_{1}, \\
& p_{3}=\beta^{*} A \varphi_{1},
\end{aligned}
$$

be the shearing force, the macrostructure, and microstructure bending moment, respectively. The Hamiltonian function is

$$
\begin{aligned}
\mathscr{H}= & \frac{p_{1}^{2}}{A(2 G-\eta)}+\frac{p_{2}^{2}}{2 E I}+\frac{p_{3}^{2}}{2 A \beta^{*}} \\
& +\frac{2 \eta \varphi+(2 G+\eta) \psi}{2 G-\eta} p_{1}+\frac{2 A \eta G(\psi+\varphi)^{2}}{2 G-\eta}+q y \\
& +m \psi+c \varphi
\end{aligned}
$$

and hence the canonical equations read (a first order system is given in (39)-(41) of [12])

$$
\begin{aligned}
y^{\prime}-2 \frac{p_{1}}{A(2 G-\eta)}-\frac{2 \eta \varphi+(2 G+\eta) \psi}{2 G-\eta} & =0, \\
\psi^{\prime}-\frac{p_{2}}{E I} & =0, \\
\varphi^{\prime}-\frac{p_{3}}{A \beta^{*}} & =0, \\
p_{1}^{\prime}+q & =0, \\
p_{2}^{\prime}+\frac{2 G+\eta}{2 G-\eta} p_{1}+4 \frac{\eta A G}{2 G-\eta}(\psi+\varphi)+m & =0, \\
p_{3}^{\prime}+\frac{2 \eta}{2 G-\eta} p_{1}+4 \frac{\eta A G}{2 G-\eta}(\psi+\varphi)+c & =0 .
\end{aligned}
$$


In this system $y$ never appears, whence (47a) may be solved independently. Indeed, (47a) and (47d) give $y$ and $p_{1}$ by a quadrature, respectively. In particular

$$
p_{1}(x)=P_{1}(x)=\bar{p}_{1}+\int_{0}^{x} q(\tau) d \tau,
$$

and $\bar{p}_{1}$ is given by the $\mathrm{BC} p_{1}(0)=\bar{p}_{1}$. Furthermore, taking the difference of (47e) with (47f), it is

$$
\left(p_{3}-p_{2}\right)^{\prime}=m-c+\frac{2 G}{2 G-\eta} P_{1}
$$

where the RHS is known. Let

$$
P(x)=\int_{0}^{x}\left(m-c+\frac{2 G}{2 G-\eta} P_{1}\right) d x+c_{0},
$$

where $c_{0}=p_{3}(0)-p_{2}(0)$. Then, the remaining equations (47b), (47c), and (47e) read

$$
\begin{aligned}
\psi^{\prime}-\frac{p_{2}}{E I} & =0, \\
\varphi^{\prime}-\frac{p_{2}}{A \beta^{*}} & =\frac{P(x)}{A \beta^{*}}, \\
p_{2}^{\prime}+4 \frac{\eta A G}{2 G-\eta}(\psi+\varphi) & =-m-\frac{2 G+\eta}{2 G-\eta} P_{1} .
\end{aligned}
$$

Such linear system can be easily reduced to a single second order linear inhomogeneous ODE in $p_{2}$; namely,

$$
\begin{aligned}
p_{2}^{\prime \prime}+4 \xi^{2} p_{2}= & -m^{\prime}-\frac{2 G+\eta}{2 G-\eta} q \\
& -4 \frac{\eta G}{(2 G-\eta) \beta^{*}}\left(m-c+\frac{2 G}{2 G-\eta} P_{1}\right),
\end{aligned}
$$

where it is let that

$$
\xi=\sqrt{\frac{\eta G\left(E I+A \beta^{*}\right)}{E I \beta^{*}(2 G-\eta)}} .
$$

The coefficient (53) corresponds to (44) of [12], although a factor $\sqrt{2}$ seems to be missing. Besides, the limit of $\xi$ as $\eta \rightarrow \infty$ appears in the first of (24) of [13], which deals with the couple stress situation. Indeed, it is observed that (23) of [13], which governs the behavior of the shearing force, is equivalent to (52), although a minus sign in front of $\xi$ appears. Such minus sign determines an exponential-type solution, which is clearly unphysical in the case of an infinite beam. The ODE (52) is the celebrated linear pendulum equation, which gives rise to oscillatory solutions. The finding of an oscillatory behavior for the couple stress and microrotation in the cantilever beam numerically investigated in is then motivated [12]. In the special case $\eta=0$, (52) reduces to the shearing force equilibrium for a Timoshenko beam. Once (52) is solved, $\psi$ and $\varphi$ may be found by a quadrature through (51a) and (51b), respectively.

\section{Conclusions}

In this paper three Timoshenko-like beam models, available in the literature, have been considered: the first model is based on the micropolar linear continuum theory of Eringen and Nowacki (E-N), the second is based on the couple stress theory of Koiter and Mindlin (K-M); and the third model is based on the modified couple stress theory proposed in [6]. A comparison of such models has been developed together with a confrontation with the original Timoshenko model. The analysis is carried out in a variational setting, making use of Hamilton's principle. It has been shown that both the Timoshenko and the couple stress model are based on a microstructural kinematics which disappears owing to it being governed by kinosthenic (ignorable) terms in the Lagrangian. Both the micropolar and the couple stress models, within a restricted beam-plane kinematics, require a single microstructural material parameter, regardless of whether the modified couple stress theory is adopted. Furthermore, it has been shown that the micropolar model formally reduces to the couple stress model upon introducing the proper constraint on the microstructure kinematics, although the material parameter is generally different (cf. [13]). Line loading on the microstructure results in a nonconservative force potential. Finally, the Hamiltonian form of the micropolar beam model has been derived together with the corresponding canonical equations. The general solution of the micropolar model has been presented, which shows a general oscillatory behavior for the microstructure rotation and couple stress. Such behavior is found in the numerical studies available in the literature.

\section{Conflict of Interests}

The author declares that there is no conflict of interests regarding the publication of this paper.

\section{Acknowledgment}

Financial support from the Fondazione Cassa di Risparmio di Modena, Pratica Sime no. 2013.0662, is gratefully acknowledged.

\section{References}

[1] A. C. Eringen, "Theory of micropolar elasticity," Tech. Rep., Princeton University, 1967.

[2] A. C. Eringen, "Part I. Polar field theories," in Continuum Physics Volume 4: Polar and Nonlocal Field Theories, pp. 1-73, Academic Press, New York, NY, USA, 1976.

[3] J. Dyszlewicz, Micropolar Theory of Elasticity, vol. 15 of Lecture Notes in Applied and Computational Mechanics, Springer, Berlin, Germany, 2004.

[4] R. D. Mindlin and H. F. Tiersten, "Effects of couple-stresses in linear elasticity," Archive for Rational Mechanics and Analysis, vol. 11, no. 1, pp. 415-448, 1962.

[5] W. T. Koiter, "Couple stresses in the theory of elasticity, parts I and II," Poceedings Koninklijke Nederlandse Akademie van Wetenschappen, Series B, vol. 67, no. 1, pp. 17-44, 1964. 
[6] F. Yang, A. C. M. Chong, D. C. C. Lam, and P. Tong, "Couple stress based strain gradient theory for elasticity," International Journal of Solids and Structures, vol. 39, no. 10, pp. 2731-2743, 2002.

[7] N. Challamel and C. M. Wang, "The small length scale effect for a non-local cantilever beam: a paradox solved," Nanotechnology, vol. 19, no. 34, Article ID 345703, 2008.

[8] S. K. Park and X.-L. Gao, "Bernoulli-Euler beam model based on a modified couple stress theory," Journal of Micromechanics and Microengineering, vol. 16, no. 11, pp. 2355-2359, 2006.

[9] I. Aganović, J. Tambača, and Z. Tutek, "Derivation and justification of the models of rods and plates from linearized three-dimensional micropolar elasticity," Journal of Elasticity. The Physical and Mathematical Science of Solids, vol. 84, no. 2, pp. 131-152, 2006.

[10] G. Riey and G. Tomassetti, "Micropolar linearly elastic rods," Communications in Applied Analysis, vol. 13, no. 4, pp. 647-657, 2009.

[11] H. M. Ma, X.-L. Gao, and J. N. Reddy, "A microstructuredependent Timoshenko beam model based on a modified couple stress theory," Journal of the Mechanics and Physics of Solids, vol. 56, no. 12, pp. 3379-3391, 2008.

[12] S. Ramezani, R. Naghdabadi, and S. Sohrabpour, "Analysis of micropolar elastic beams," European Journal of Mechanics, A/Solids, vol. 28, no. 2, pp. 202-208, 2009.

[13] M. Asghari, M. H. Kahrobaiyan, M. Rahaeifard, and M. T. Ahmadian, "Investigation of the size effects in Timoshenko beams based on the couple stress theory," Archive of Applied Mechanics, vol. 81, no. 7, pp. 863-874, 2011.

[14] S. P. Timoshenko and J. M. Gere, Theory of Elastic Stability, Dover Publications, 2nd edition, 1961.

[15] C. Lanczos, Variational Principles of Mechanics, Dover, 1986.

[16] A.Nobili, "Variational approach to beams resting on two-parameter tensionless elastic foundations," Transactions ASMEJournal of Applied Mechanics, vol. 79, no. 2, Article ID 021010, 2012.

[17] A. Nobili, "Superposition principle for the tensionless contact of a beam resting on a winkler or a pasternak foundation," Journal of Engineering Mechanics, vol. 139, no. 10, pp. 1470-1478, 2013.

[18] A. Nobili and A. M. Tarantino, "Magnetostriction of a hard ferromagnetic and elastic thin-film structure," Mathematics and Mechanics of Solids, vol. 13, no. 2, pp. 95-123, 2008.

[19] A. Nobili and L. Lanzoni, "Electromechanical instability in layered materials," Mechanics of Materials, vol. 42, no. 5, pp. 581$591,2010$.

[20] A. Nobili and A. M. Tarantino, "Pseudo-spectral methods in one-dimensional magnetostriction," Meccanica, vol. 50, no. 1, pp. 99-108, 2015.

[21] C. M. Wang, J. N. Reddy, and K. H. Lee, Shear Deformable Beams and Plates: Relationships with Classical Solutions, Elsevier, 2000. 


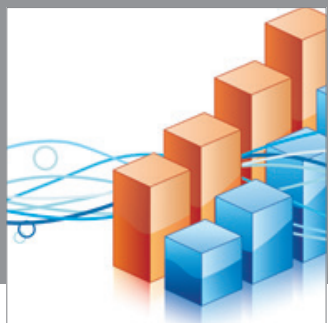

Advances in

Operations Research

mansans

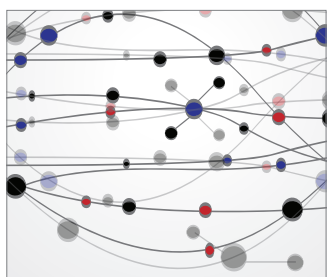

The Scientific World Journal
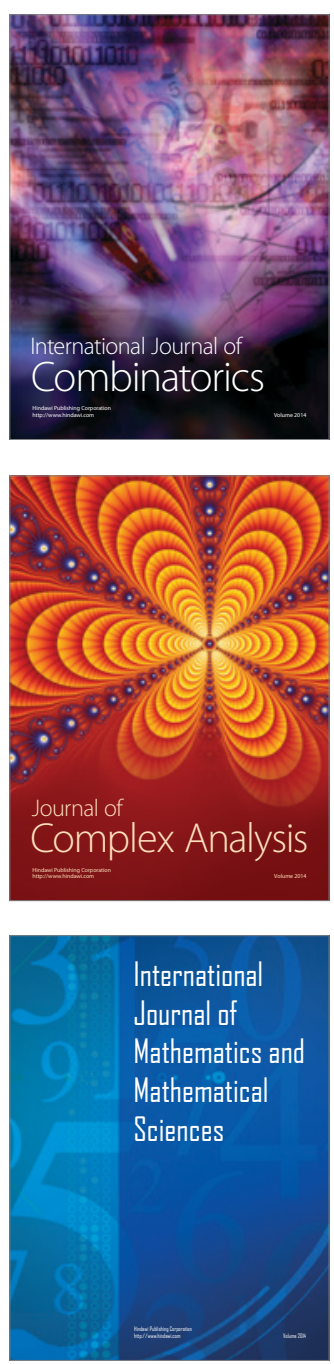
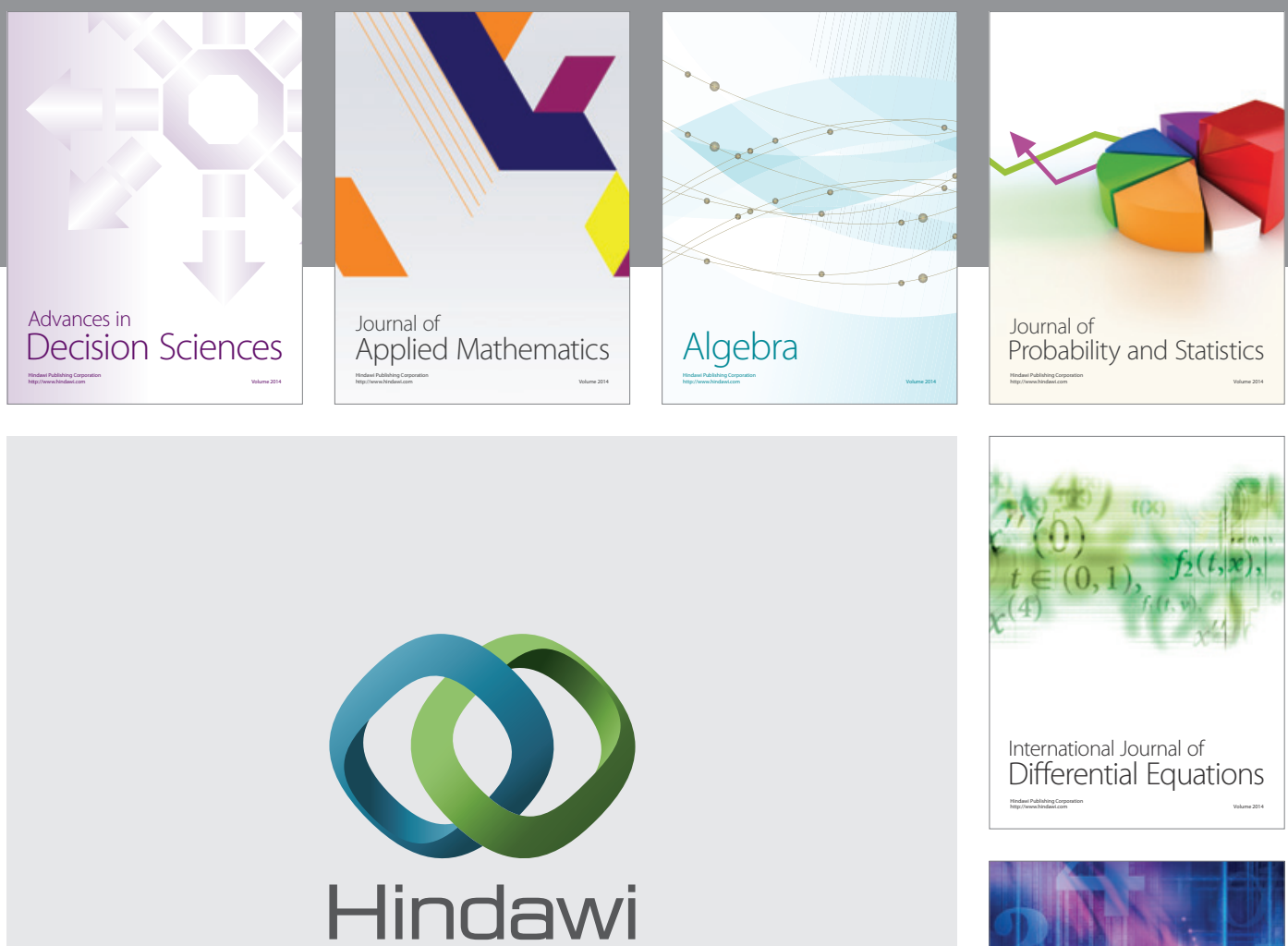

Submit your manuscripts at http://www.hindawi.com
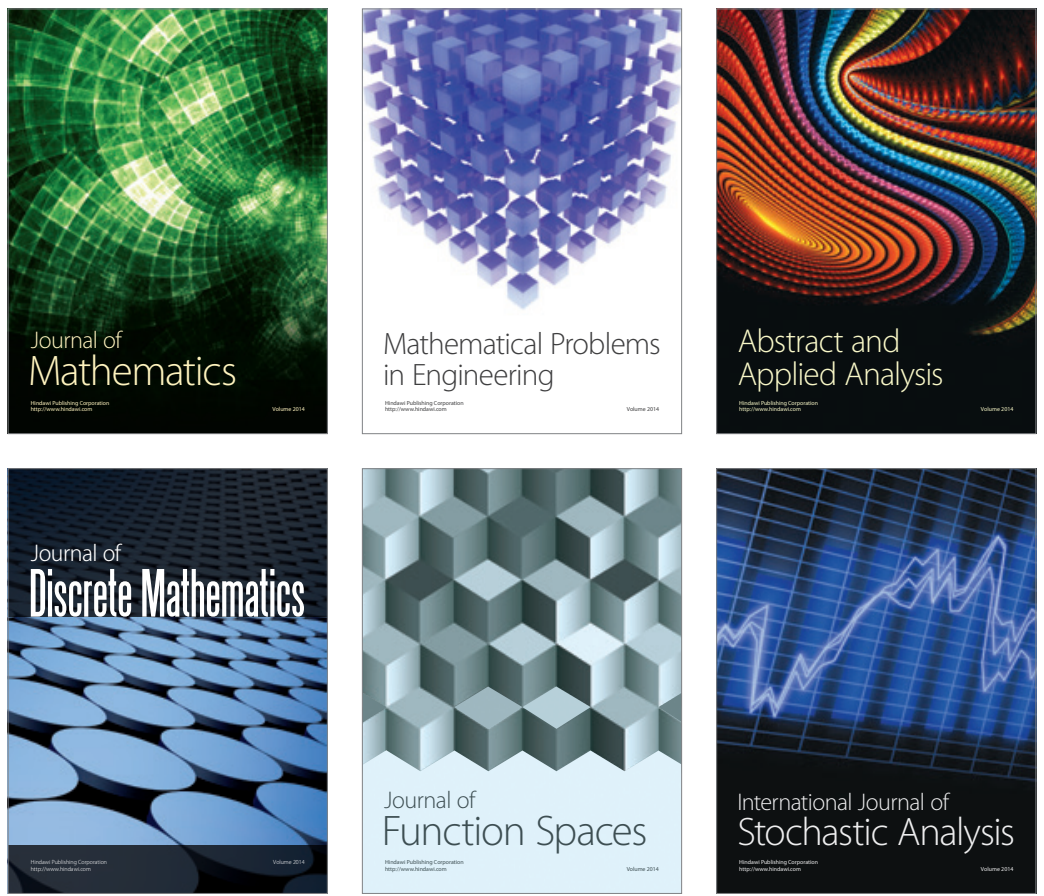

Journal of

Function Spaces

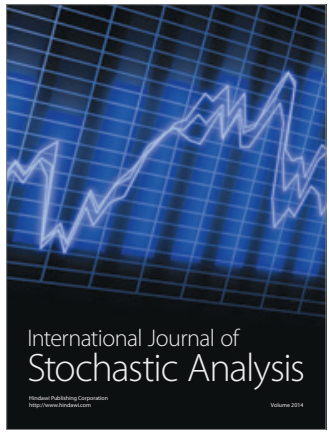

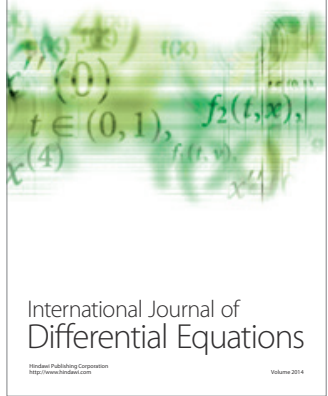
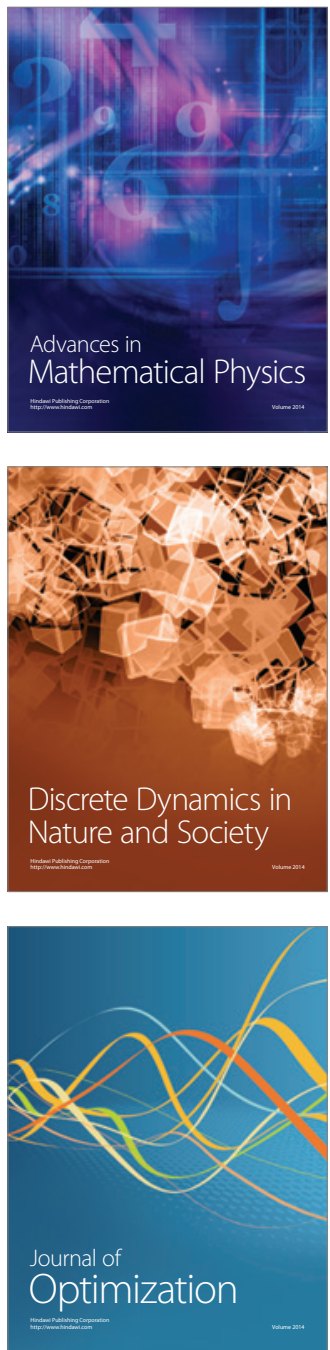